\title{
ANOTHER PROOF OF THE MINIMAX THEOREM
}

\section{J. NEWMAN}

There are many known proofs of the fundamental theorem of 0 -sum, 2 person game theory, the so-called minimax theorem. The following proof, however, seems to be the shortest yet.

If $x=\left(x_{1}, x_{2}, \cdots, x_{N}\right)$ is a vector in $E^{N}$, then we write, as usual, $x \geqq 0$ to mean $x_{i} \geqq 0, i=1, \cdots, N, x \geqq 0$ to mean $X \geq 0$ and $X \neq 0$. In what follows $M$ denotes an arbitrary fixed real $m \times n$ matrix and $J$ denotes the $m \times n$ matrix all of whose entries are $1 . M^{T}$ denotes, as usual, the transpose of $M$. Consider now,

1. Minimax Theorem. There exists a real number $\nu$ such that $(M-\nu J) x \geqq 0$ for some $x \in E^{m}, x \geq 0$ and $\left(-M^{T}+\nu J^{T}\right) y \geqq 0$ for some $y \in E^{n}$ with $y \geq 0$.

2. Theorem of the Alternative. Either

$$
M x \geqq 0 \text { for some } x \in E^{m}, x \geq 0,
$$

or

$$
-M^{T} y \geqq 0 \text { for some } y \in E^{n}, y \geq 0 .
$$

3. Stiemke's Theorem [1]. If $S$ is a subspace of $E^{N}$ and $S^{\perp}$ is its orthogonal complement, then $S \cup S^{\perp}$ contains some vector $X$ with $X \geqq 0$.

We shall prove 3 and $3 \rightarrow 2 \rightarrow 1$ (although the proofs of 3 and $2 \rightarrow 1$ are standard we include them for completeness).

Proof of 3. Let $A$ be the (closed) set of all vectors $x \in E^{N}$ such that $|x| \geqq 1, x \geqq 0$. Let $P$ be the operator of projection onto $S$, call $B=P(A)$ and let $y=P(z)$ be a vector in $B$ of minimal length. Suppose $y=\left(y_{1}, y_{2} \cdots y_{n}\right)$ had some negative component, say $-y_{i}$, then, with $w=\left(0,0, \cdots, y_{i}, 0, \cdots\right),|y+w|<|y|$, and so $|P(z+w)|$ $=|y+P(w)| \leqq|y+w|<|y|$, and this is a contradiction since $Z+W$ clearly lies in $A$. Hence $y \geqq 0$. If $y=0$ then $z \in S^{\perp}$ and the result follows since $z \in A$. If $y \geq 0$ then the result follows since $y \in S$.

We now need the following

Definition. If $\left(z_{1}, z_{2}, \cdots, z_{m}\right)=Z \in E^{m}$ and $\left(w_{1}, w_{2}, \cdots, w_{n}\right)$ $=w \in E^{n}$ then the vector in $E^{m+n}$ given by

$$
\left(z_{1} z_{2}, \cdots z_{m}, w_{1}, w_{2}, \cdots w_{n}\right)
$$

will be denoted by $z \times w$.

Received by the editors October 24, 1959 and, in revised form, January 4, 1960. 
Proof that $3 \rightarrow 2$. It is easily seen that the set of all vectors of the form $x \times M x, x \in E^{m}$, forms a subspace of $E^{m+n}$, as does the set of all vectors of the form $-M^{T} y \times y, y \in E^{n}$. Next note that these subspaces are in fact orthogonal complements in $E^{m+n}$. An application of 3 tells us that either $x \times M x \geqq 0$ for some $X \in E^{m}$ (in which case $x \geq 0, M x \geqq 0$ ) or that $-M^{T} y \times y \geqq 0$ for some $y \in E^{n}$ (in which case $\left.y \geq 0,-M^{T} y \geqq 0\right)$. In either case 2 is verified.

Proof that $2 \rightarrow 1$. Let $S_{1}$ be the set of all real numbers, $\nu$, for which $(M-\nu J) x \geqq 0$ for some $x \geqq 0$ and similarly let $S_{2}$ be the set of $\nu$ for which $\left(-M^{T}+\nu J^{T}\right) y \geqq 0$ for some $y \geqq 0$. It follows directly that both $S_{1}$ and $S_{2}$ are closed. Neither $S_{1}$ nor $S_{2}$ are void since $S_{1}$ contains all large negative numbers while $S_{2}$ contains all large positive numbers. Applying 2 to the matrix $M-\nu J$ tells us that every $\nu \in S_{1} \cup S_{2}$. Connectedness of the line now implies that $S_{1}$ and $S_{2}$ must overlap, and this is the statement 1 .

\section{REFERENCE}

1. A. W. Tucker, Extensions of theorems of Farkas and Stiemke, Abstract 76, Bull. Amer. Math. Soc. vol. 56 (1950) p. 57.

BROWN UNIVERSITY AND

Sylvania Electronics, Needham, Massachusetts 